\title{
Rare Quadricuspid aortic valve with acute early incapacitation
}

\author{
Geoffrey Joseph Changwe ${ }^{1}$, Haiyang $W^{2}{ }^{2}$, Chengwei Zou ${ }^{2}$, and Gang Zhang ${ }^{2}$ \\ ${ }^{1}$ Shandong University School of Medicine \\ ${ }^{2}$ Shandong Provincial Hospital
}

October 9, 2020

\begin{abstract}
Abstract A 39-year-old male patient from a county health facility presented to our department with a one-year history of progressive exertional chest tightness (NYHA class III-IV), which resolved completely within five to six minutes of rest. Both motion and static imaging results revealed a rare solitary Quadricuspid aortic valve (QAV) with severe aortic valve regurgitation. The QAV was replaced with mechanical prosthetic valve via mini-superior sternotomy on cardiopulmonary bypass machine. The patient was reviewed a month after operation, and assessment revealed that he had reverted to NYHA class-I. A rare congenital lesion such as QAV, repair may not be the best option due to lack of long-term data on longevity. Additionally, choice of a mechanical prosthetic valve guarantees unwanted re-operations associated with possible failure of bioprosthetic valves.
\end{abstract}

\section{Introduction}

Quadricuspid aortic valve (QAV) is an exceedingly rare finding with a little more than 200 cases reported since 1862[1]despite recent advancement in cardiac imaging modalities. Although QAV has been reported in subjects presenting with no clinically significant symptoms, aortic insufficiency (of variable degree) due to malcoaptation, and subsequent complications remain the hall-mark clinic manifestation usually noted during the $4^{\text {th }}$ decade and thereafter [2]. Case presentation

A 39-year-old male patient with no history of smoking was admitted to our department with a one-year history of progressive exertional chest tightness, which resolved after five to six minutes of resting. Chest auscultation uncovered a diastolic murmur at Erb's point, while electrocardiogram performed at rest revealed no conduction abnormalities. His vitals measured as follows; pulse 68; respirations 17 and systolic blood pressure 132/72 mmHg. Transthoracic echocardiography during initial examination uncovered a QAV with severe regurgitation due to malcoaptation. Although presentation mimic coronary artery diseases, coronary angiography was not performed due to low suspicion index. Instead, computed tomography was performed to confirm the QAV structure and exclude associated cardiac lesions. The patient was scheduled for urgent elective aortic valve replacement.

Prior to valve replacement, intraoperative transoesophageal echocardiography was performed to delineate the following cardinal structures: aortic root, Sinus of Valsalva and Sino-tubular junction diameters, which measured $: 2.92 \mathrm{~cm}, 4.40 \mathrm{~cm}$ and $3.48 \mathrm{~cm}$ respectively. Additionally, the ejection fraction and the left ventricular end diastolic dimensions measured $: 57 \%$ and $7.1 \mathrm{~cm}$ respectively. The static QAV imaging and intraoperative results are shown in (figure.1), and motion imaging results in (video.1 and video.2).

\section{Discussion}

Despite recent advancement in imaging modalities, the incident of QAV, either alone or with associated malformations has remained exceedingly a rare finding $[1,2]$. The categorization of QAV based on structure has been well documented by earlier pioneers [3]. Commonly, QAV is incidentally found amongst clients who present with diastolic murmur as the only chief concern to seek medical attention. On the other 
hand, QAV can present with various symptoms, however, central-jet aortic valve regurgitation predominates. Complication of aortic regurgitation due malcoaptation of QAV include: dilatation of aortic root, Sinus of Valsalva and ascending aorta. Others include significant enlargement of left ventricle and left atrium with subsequent conduction abnormalities which include either atrial fibrillation or RBBB [3,4].

Our reported case is notably peculiar and worthy reporting not because of the rareness of QAV alone, but the inability to elicit significant cardiac chamber and great vessel enlargement despite one-year duration of progressive exertional chest tightness (NYHA class-III-IV). The progressive reduction in ejection fraction in such a young and supposedly energetic young man rendered him incapacitated, as he was unable to participate in strenuous activities. Additionally, untimely valve replacement risked severe and sudden global hypoperfusion during strenuous activities with subsequent sudden death [4]. The unpredictability of age at which QAV may manifest severe clinical symptoms despite insignificant great-vessel and cardiac remodeling underscores the incomprehensive study of QAV at molecular level due to its rareness.

Similar to other facilities world over, due to patients age we opted to replace the native valve with a mechanical prosthetic valve via open heart surgery and avoid repeat of surgery due to bioprosthetic valve deterioration $[5,6]$. Although repair of native QAV has been reported, our facility favored replacement due to lack of reliable data on the longevity of repaired cases, and avoid unwanted repeat of surgery. A month post-surgery, the patient was reviewed and had reverted to (NYHA class-I). He was maintained on life-long anticoagulants with reviews scheduled at his local health facility.

\section{Acknowledgement: none.}

Funding: none

Conflict of interest ; none declared.

Patient's consent : written consent obtained.

Institutional Ethics Committee approval : waiver obtained.

References

[1.] Godefroid O, Colles P, Vercauteren S, Louagie Y, Marchandise B. Quadricuspid aortic valve: A rare etiology of aortic regurgitation. Eur J Echocardiogr. 2006; 2:168-70.

[2]. Choji CL, Selvaraj N, Prather J. Female Adolescent with Quadricuspid Aortic Valve. J Am Osteopath Assoc. $2015 ; 115(9): 570$.

[3]. Hurwitz LE, Roberts WC. Quadricuspid semilunar valve. Am J Cardiol. 1973;31:623-6.

[4]. Tsang MYC, Abudiab MM, Ammash NM, Naqvi TZ, Edwards WD, Nkomo VT and Pellikka PA. Quadricuspid Aortic Valve: Characteristics, Associated Structural Cardiovascular Abnormalities, and Clinical Outcomes. Circulation. 2016; 133:312-9.

[5]. Yuan S-M, Song-Li Y. Quadricuspid aortic valve: A case report. Cor Vasa. 2016; 5:8513-4.

1. Head SJ, Çelik M, Kappetein AP. Mechanical versus bioprosthetic aortic valve replacement. European Heart Journal. 2017;38:2183-91.

Figure legends:

1.Figure.1. Imaging results: A, B and D; Transoesophageal echocardiography (TEE) short axis view showing Quadricuspid aortic valve structure(3-D) and regurgitation. E, TEE long axis view showing severe regurgitation. C; Computed tomography showing valvular structure, and F; intraoperative findings.

2. Transoesophageal echocardiography motion showing valve structure and regurgitation;

Video.1 and Video.2. 

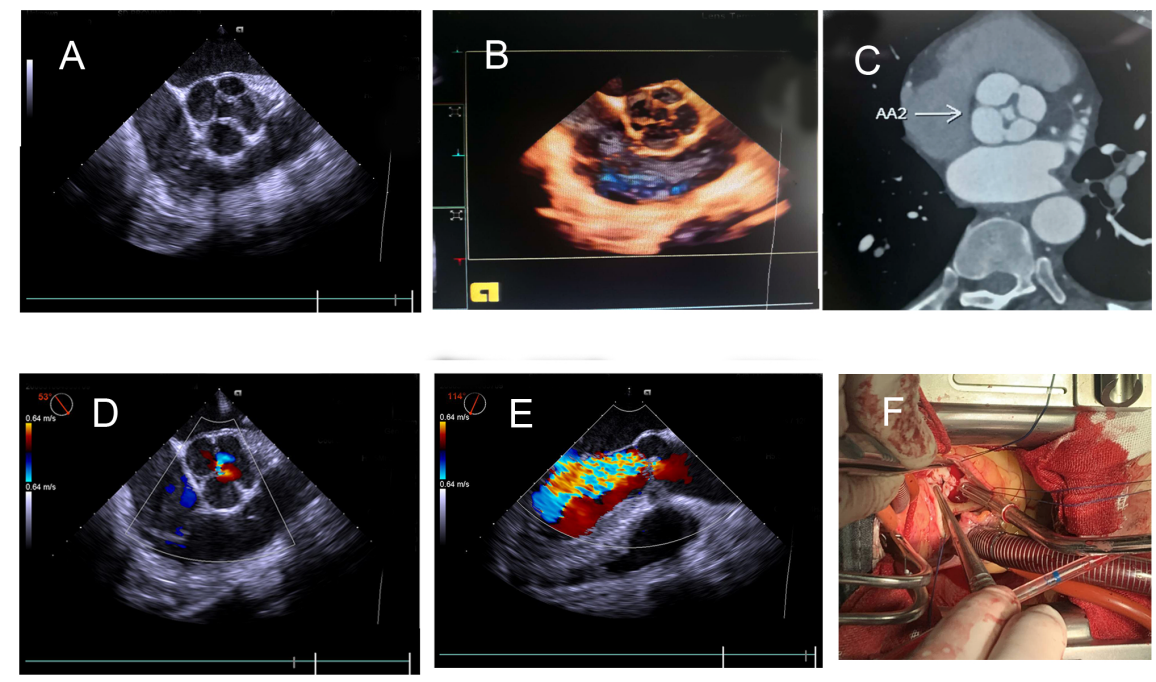